\title{
Grenzen, Körper und Gewalt: Wie Pornografie die Grenze zwischen Mexiko und den Vereinigten Staaten erzählt ${ }^{1}$
}

\section{Borders, Bodies, and Violence: How Pornography Narrates the Border Between Mexico and the United States}

\section{Anna Casaglia, Trient}

Zusammenfassung: Dieser Artikel problematisiert das pornografische Genre des „Border Sex“, welches an der Grenze zwischen den USA und Mexiko spielt und in den USA produziert wird und welches ungleiche Machtverhältnisse darstellt, die an der Grenze und durch die Grenze Gestalt annehmen. Ich entwickle dabei eine Interpretation dieser Darstellungen, indem ich auf den Einsatz von institutionalisierter Gewalt als Mittel zur Ausübung von Kontrolle über die Körper von Migrantinnen fokussiere. Meine Interpretation hebt die intersektionalen Aspekte von Ungleichheiten hervor und erarbeitet ein Verständnis der Verkörperung von Kontrolle und der Grenze zwischen den USA und Mexiko, welches die Bedeutung und das Potenzial der theoretischen und analytischen Werkzeuge der feministischen Geographie aufzeigt, um so einen Beitrag zur kritischen Grenzregimeforschung zu leisten.

Schlagwörter: Grenzregime; Grenzkontrollen; Macht; Gewalt; Intersektionalität; Pornografie; feministische Geographie; USA-Mexiko

Abstract: This article problematizes the pornographic genre of "border sex" set on the U.S.-Mexico border and produced in the United States, which depicts unequal power relations that take shape at the border and through the border. In doing so, I

1 Der Originalbeitrag „Confini, corpi e violenza: come la pornografia racconta la frontiera tra Messico e Stati Uniti“ ist in Smagliature Digitali. Corpi, Generi, Tecnologie, hrsg. von Carlotta Cossutta et al. (Agenzia X, 2018), 119-132, erschienen. Übersetzung von Andreas Oberprantacher. 
develop an interpretation of these representations by focusing on the use of institutionalized violence as a means of exerting control over immigrant women's bodies. My interpretation highlights the intersectional aspects of inequalities and elaborates an understanding of the embodiment of control and the U.S.-Mexico border that demonstrates the importance and potential of the theoretical and analytical tools of feminist geography to contribute to critical border regime research.

Keywords: border regimes; border control; power; violence; intersectionality; pornography; feminist geography; U.S.-Mexico.

Rule 34: "There is porn of it, no exception"

Rule 35: "If no porn is found of it, it will be made"

Rule 36: "There will always be more fucked up shit than what you just saw"

(www.4chan.org)

Wenn etwas existiert, gibt es eine Pornoversion davon. In den letzten Jahren hat sich mehr denn je gezeigt, dass Pornografie, auch dank immer kürzerer Produktions- und Vertriebszeiten, in der Lage ist, Ereignisse und Veränderungen in der Gesellschaft pünktlich einzufangen, sie in ein erotisches Imaginäres zu integrieren und $\mathrm{zu}$ einer sexzentrierten Parodie $\mathrm{zu}$ formen. Geopolitische Phänomene sind vor dieser Regel nicht gefeit, auch das Thema der Migration und ihrer Kontrolle nicht, welches auf der Tagesordnung von westlichen Ländern steht und diese beunruhigt.

In diesem Text werde ich mich auf die kritische Analyse eines pornografischen Genres namens Border Patrol Sex konzentrieren, das an der Grenze zwischen Mexiko und den Vereinigten Staaten angesiedelt ist und die Formen der Überwachung sowie die diversen Machtverhältnisse, die sich an der Grenze abspielen und realisieren, inszeniert. Neben der Analyse der geopolitischen Repräsentation der Grenze interessiert mich auch die Rolle, die lateinamerikanische Migrantinnen in diesen Videos spielen, und die Art und Weise, wie die Protagonistinnen durch diese Repräsentation die Grenze verkörpern; denn Grenzkontrolle impliziert auch eine Kontrolle von Körpern. Das Interesse, das dieser Forschungsarbeit zugrunde liegt, betrifft daher die Frage, wie die Darstellung von Grenzen und lateinamerikanischen Migrantinnen in dieser Art von Pornovideo eine Möglichkeit bieten kann, 
den Zusammenhang zwischen Grenzen, Mobilität, Körpern und Gewalt zu analysieren und neu zu imaginieren.

Mein Interesse an der Pornografie und am subversiven Potenzial bestimmter Inhalte im Hinblick auf den hegemonialen heteronormativen Diskurs über Sex rührt vor allem von meiner persönlichen und politischen feministischen Erfahrung her sowie vom Durchlaufen der Post-Porno-Bewegung, aus der meine Neugier für Pornografie als umstrittenes Objekt erwächst. Eine Überlegung, die ich für wichtig erachte, betrifft meine Positionalität als westliche, weiße Frau bei der Durchführung dieser Forschung, was einerseits eine große Schwierigkeit bei der Behandlung bestimmter Themen und insbesondere bei der Wahl des Analyseobjekts darstellt, andererseits aber auch die Anerkennung eines offensichtlichen Privilegs impliziert, durch das diese Thematiken beobachtet und analysiert werden können.

Als Grenzforscherin interessiere ich mich für Erfahrungen, die täglich im Umgang mit Grenzen gemacht werden, d.h. für die Art und Weise, wie sie heute überall zugegen sind und das Leben der Menschen durchdringen und bedingen, selbst in Kontexten und Situationen, die von der eigentlichen Grenze - verstanden als eine Barriere, die zwei Nationalstaaten trennt weit entfernt wären. So ein Zugang erfordert die Überwindung der Vorstellung von der Grenze als einer Linie auf dem Territorium (Parker und Vaughan-Williams 2012), um stattdessen die Art und Weise zu erörtern, wie das so genannte Borderwork, so wie es von Rumford (2012) definiert wird, in der täglichen Erfahrung der Menschen wirkt. Die Grenze ist eine diskursive Landschaft, in der eine normative Dimension und Alltagserfahrungen koexistieren. Über ihre Durchdringung nachzudenken (Balibar 2002) bedeutet auch, sie als eine Technologie zu analysieren, die soziale Spaltungen reproduziert, etwa durch die Kontrolle und Disziplinierung von Körpern.

Eine Möglichkeit, diese Ebene der Analyse zu verstehen, besteht darin, populäre Kulturprodukte und die Art und Weise zu untersuchen, wie sie Grenzen und die mit ihnen verbundenen Phänomene beschreiben, darstellen, reproduzieren und debattieren. Ein erster Schritt in diese Richtung ist die Anerkennung der wesentlichen Verbindung zwischen Geographie und Macht (Ó Tuathail 1996) und zwischen Geographie und Diskurs. Innerhalb der kritischen politischen Geographie hat sich eine geopolitische Analyse kultureller Produkte entwickelt, welche, ausgehend von diesen Verbindungen, verstehen will, wie Macht durch die Medien repräsentiert und reproduziert wird (Dittmer und Dodds 2008; dell'Agnese und Amato 2016; Coletti 2018). Kulturelle Produkte und, mehr noch, Pop-Produkte beeinflussen un- 
ser geographisches und geopolitisches Imaginäres, indem sie der Welt um uns herum Bedeutungen hinzufügen. Die durch Bilder vermittelten Inhalte werden Teil unseres Bedeutungsgepäcks und eben deshalb wird es interessant, die Populärkultur zu dekonstruieren, um diese Bilder zu entdecken und um zu verstehen, wie sie dazu beitragen könnten, spezifische Diskurse zu bestimmen, zu verstärken oder zu konterkarieren.

Dasselbe gilt für die Inszenierung der Subjekte und deshalb ist es von wesentlicher Bedeutung, dass sich kritisches Denken, wie Butler (2004, 140) vorschlägt, auf die Repräsentation als einen jener Hauptbereiche konzentriert, in denen Praktiken der Vermenschlichung und Entmenschlichung unaufhörlich hervorgebracht werden. Wer der Möglichkeit der Selbstdarstellung beraubt ist, wird auch leichter vom Privileg ausgeschlossen, durch die Darstellungen anderer oder deren Verneinung vermenschlicht zu werden (ebd.).

Es ist von Bedeutung, die Pornografie als ein eigenes Studienobjekt zu behandeln, als ein populäres Genre, dessen Inhalte, Darstellungen, Emotionen und fleischliche Resonanzen (Paasonen 2011) es wert sind, analysiert zu werden, auch im Hinblick auf ihre Verbreitung und Fähigkeit, ein erotisches Imaginäres zu orientieren und zu beeinflussen. Die Darstellung von Sex findet in der Pornografie nicht in einem raum-zeitlichen Vakuum statt, sondern ist immer, oder fast immer, historisch, geographisch und sozial bedingt. Folglich können Pornofilme und -videos auch als kulturelle Produkte analysiert werden, die nicht nur Sexualität, sondern auch bestimmte Diskurse und Darstellungen der Gegenwart reproduzieren und/oder infrage stellen.

Die Mainstream-Darstellung von Sex wurde nicht nur im feministischen Bereich stark kritisiert, weil sie ein erotisches Imaginäres fernab jeglicher Realität, stark chauvinistisch und stereotypisiert, wiederholt. Es gibt Formen der alternativen Pornografie, die diese Darstellung zur Diskussion stellen, Körper und Genitalien zeigen, welche der vorherrschenden ästhetischen Norm zufolge nicht „perfekt“ sind, eine nichtperformative Sexualität inszenieren, Frauen und ihren Begehren eine Stimme geben und diese so aus den stereotypisierenden Kategorien der Pornografie heraustreten lassen. Tatsache bleibt, dass der Mainstream-Porno seinem Wesen nach explizit ist und auf Konventionen sowie einer viszeralen und oft verzweifelten Darstellungsweise beruht (ebd.): Rassenstereotype beispielsweise waren Gegenstand von Studien, die verdeutlichen, wie Andersartigkeit, d.h. nicht weiß-westlich zu sein, fast immer mit einer wilden Hyper-Sexualität oder irgendeiner Form von „Perversion“ assoziiert wurde - hier zu verstehen als Abweichung von der Norm des gesunden Menschenverstandes. 
Grenzen und andere geopolitische Phänomene sind in ihrer populären Darstellung in Filmen, Literatur, Comics und anderen kulturellen Produkten weitgehend analysiert worden. Die Pornografie hingegen hat bei den Erforscher*innen der populären Geopolitik bisher relativ wenig Aufmerksamkeit erregt. Sie war vor allem innerhalb der Kulturwissenschaften Gegenstand der Analyse (Williams 2004; Paasonen 2011), welche über die rechtlichen, politischen und moralischen Aspekte hinausgehend ihre Inhalte im Hinblick auf verschiedene soziale und kulturelle Fragen analysieren, von der Reproduktion von Geschlechter- oder ethnischen Stereotypen angefangen über die Art und Weise ihrer Verwendung bis hin zu den Dynamiken von Produktion und Verteilung und noch mehr. Die Aufforderung, die „Pornografie ernst zu nehmen“(Williams 2004, 5), entspringt der Erkenntnis, dass es sich um eine zunehmend verbreitete Kulturform handelt, auch und vor allem dank der digitalen Produktion und Online-Verbreitung, die auf freie und anonyme Weise sowie mittels einer unendlichen Vielfalt an Nischen, Stilen und Formaten auf die Vorstellungskraft verschiedenster Menschen einwirkt.

Die Grenze, auf die sich diese Analyse konzentriert, ist die Grenze zwischen Mexiko und den Vereinigten Staaten, die, wie mehrere Autor*innen gezeigt haben (siehe z. B. Holtzman 2000, dell'Agnese 2005, 2015), in der Populärkultur schon länger sehr stark vertreten war und in dieser medialen Überbelichtung immer wieder erfunden und neu erfunden wurde, was zur Folge hatte, dass eine Reihe von Stereotypen und Gemeinplätzen entstand, die untrennbar mit dieser Grenze und der Idee, die wir von ihr haben, verbunden sind. Die geopolitische Analyse der Darstellung der Grenze zwischen Mexiko und den Vereinigten Staaten kann, auf welche Art von Medien sie sich auch immer bezieht, nicht umhin, die Konnotationen zu beachten, die dem, was auf der einen und auf der anderen Seite der Grenze liegt, beigemessen werden. Die Charakterisierung der beschriebenen Orte, Personen und Situationen ist nichts anderes als der populäre Ausdruck eines geopolitischen Diskurses über die Rolle der Vereinigten Staaten in Bezug auf Mexiko (dell'Agnese 2005, 206). An der Grenze wird es unmöglich, die Machtungleichheiten zu ignorieren, welche die Beziehungen, die dort stattfinden, strukturieren und bedingen (Donnan und Magowan 2010, 94). Diese Ungleichheiten sind wiederum Ausdruck von Machtverhältnissen, die mit „kolonialen Phantasien“ (dell'Agnese 2005, 205) einhergehen und sich in ethnischen und geschlechtsspezifischen Stereotypen übersetzen.

Grenzen zu überschreiten bedeutet oft, die eigenen Limits zu überschreiten, zu reifen, Neues zu entdecken, und die Bilder, die diese Über- 
schreitung begleiten, sind oft mit einer Idee von Verführung verbunden. In der Populärkultur symbolisieren Bilder der Grenzüberschreitung die Überschreitung sexueller Limits und eröffnen das Versprechen erotischer Emanzipation und Erfüllung. Diese Bilder spiegeln und reproduzieren - mehr oder weniger explizit - ein Ungleichgewicht von Reichtum und Macht zwischen den Menschen, die auf der einen oder auf der anderen Seite der Grenze stehen, und sind um Grenzen herum strukturiert (Donnan und Magowan 2010).

Die Grenze zwischen den Vereinigten Staaten und Mexiko wurde im 20. Jahrhundert mehr noch als jede andere Grenze als Quelle sexueller Erregung dargestellt. Ihre Beschreibung weist immer Merkmale von Korruption, leichtem Sex und berüchtigten Bars auf, in denen man sich allem hingeben kann, was nördlich der Grenze gesetzlich verboten ist und/oder von der Gesellschaft sanktioniert wird. In der nordamerikanischen Populärkultur haben Bücher, Filme, Comics und Zeichentrickfilme dazu beigetragen, ein Imaginäres zu schaffen, in dem die Grenze „einen reinen von einem unreinen Körper, einen tugendhaften von einem sündigen Körper, einen monogamen Körper, der dem Gesetz der Ehe unterliegt, von einem unanständigen, der sich der Unzucht, dem Ehebruch, der Prostitution, der Bestialität und der Sodomie hingibt“ (Gutiérrez 1996, 255-256, Übersetzung der Autorin), trennt.

Seit den 1990er Jahren und mit noch größerer Intensität nach dem Angriff auf die Zwillingstürme im Jahr 2001 hat die Südgrenze der Vereinigten Staaten einen beispiellosen Prozess der Versicherheitlichung und Militarisierung durchlaufen (Cornelius 2005) und ist auch für andere Länder und andere Grenzen zu einem „Modell“ der Kontrolle und Zurückweisung geworden. Die Entwicklung zum Management von Migrationsprozessen hat sowohl Formen der Auslagerung der Kontrolle als auch die interne Versicherheitlichung gemäß der Formel detection, detention and deportation (Menjívar 2014, 360) mit sich gebracht. Der Bau der Mauer hat die Migrationsgeographien verändert und zwingt die Routen in die Wüstengegend im Süden Arizonas, deren Überquerung sehr gefährlich und oft tödlich ist (Cornelius 2005; Aquino 2012). Die Null-Toleranz-Strategie im Umgang mit den Migrationsströmen in die Vereinigten Staaten ist auch zum Gegenstand einer populären Darstellung geworden, welche die sicherheitspolitische Paranoia reproduziert, verstärkt und zugleich die Nationalhelden, die das Land vor illegaler Einwanderung und Terrorismus verteidigen, bejubelt. 
Die Pornografie hat diese doppelte Seele der Grenze - Verführung und Sicherheit - zu einer Einnahmequelle verwandelt, dank Videos, die angebliche „illegale Latinas“ (so werden sie dargestellt) in einer Pluralität von Situationen zeigen, die verschieden sind, aber sich wiederholen, und in denen Männer die Uniform der Grenzbeamten des U.S. Immigration and Naturalization Service tragen. Konkret konzentrierte sich meine Analysearbeit auf die Website Borderpatrolsex.com, die Videos von jungen Frauen vermarktet, welche angeblich beim Versuch, die Grenze illegal zu überqueren, abgefangen wurden und denen Sex als Alternative zur Abschiebung angeboten oder von ihnen erzwungen wird. Diese in einer nicht näher spezifizierten Wüstenumgebung gedrehten Videos reproduzieren immer wieder dieselbe Struktur: In einem für Pornos sehr artikulierten Vorspann werden die täglichen Patrouillen der Grenzschutzbeamten wiedergegeben, wobei über ihre Arbeit, die verfügbare Ausrüstung und die Identifizierung illegaler Migrant*innen in der Wüste gesprochen wird. Während ihres Dienstes entdecken sie Migrantinnen oder Gruppen von Männern und Frauen ohne Papiere, die manchmal bewaffnet und/oder im Besitz von Drogen sind. An dieser Stelle können die Handlungsstränge voneinander abweichen, wobei ein konstantes Element durch die Tatsache gegeben ist, dass die beiden Hauptprotagonist*innen, ein Wächter und eine lateinamerikanische Frau, isoliert werden. In weiterer Folge kommt es zu Sex, entweder in Form einer Erpressung, d.h. auf mehr oder weniger gewaltsame Weise, oder als Ergebnis von Verhandlungen. Ist Ersteres der Fall, wird die Frau am Ende der Sexszene regelmäßig auf einen Jeep geladen, um abgeschoben zu werden, während im zweiten Fall das Ende offen ist für Drehungen und Wendungen.

Die Serie, die von der Produktionsfirma $M_{o f o s}{ }^{2}$ erschaffen wurde, wird auf der gleichnamigen Website als Gelegenheit für den Zuschauer beschrieben, die Agenten Smith und Martinez zu begleiten, wie sie in der Wüste auf der Suche nach „hot border jumpers“ patrouillieren. Zusätzlich zu den Videos machen die weiteren Inhalte auf der Seite (Fotogalerien und Text) deutlich, dass es neben dem Sex noch andere relevante Elemente gibt, welche diese Serie charakterisieren, was etwa in einer Rezension betont wird, die auf einer Website veröffentlicht wurde, welche mehrere Pornokanäle präsentiert und kommentiert:

2 Mofos ist ein 2008 gegründetes kanadisches Produktionsunternehmen, das sich im Besitz der Vertriebsgruppe Mindgeek befindet. 
Die Geschichte dieser Seite [borderpatrolsex.com] ist einfach und sehr erregend. Die Border-Patrol-Agenten patrouillieren an der Südgrenze des Landes. Sie verfügen über moderne Ausrüstungsgegenstände wie Radar, Nachtsichtferngläser, Quads und selbstverständlich Jeeps. Sie stehen über Funk miteinander in Kontakt und koordinieren Operationen. Dank dessen sind sie sehr effektiv und es ist nicht leicht, illegal ins Land einzureisen. Wie Sie wissen, stellt der Zustrom illegaler Einwanderer*innen eine Bedrohung für die Wirtschaft dieses Landes dar. Deshalb ist die Arbeit der Border Patrol so wichtig. (www.sexpilot.org; Übersetzung d. Autorin)

Im Moment ist die Website aus Gründen geschlossen, die ich bisher nicht überprüfen konnte, aber ich würde nicht ausschließen, dass die Entscheidung auch wegen ihrer spezifischen Inhalte getroffen wurde, welche in einigen Blogs und Websites infolge der Art und Weise kritisiert wurden, wie sie das Leiden von Migrantinnen ausbeuten, zumal der Kanal Anfang 2014 gestartet wurde, d.h. zu einer Zeit, als es in den Vereinigten Staaten Untersuchungen und Nachrichten bezgl. des sexuellen Missbrauch von Migrantinnen durch Grenzschutzbeamte gab. Die Hauptkritik an dieser Serie besteht darin, dass sie das Trauma, das Migrantinnen erleiden, zu einer Quelle der Erregung macht ${ }^{3}$ und die Realität auf ekelhafte Weise nachahmt. ${ }^{4}$ Trotz der Schließung der Website sind fast alle Videoinhalte weiterhin über jedes Pornoportal zugänglich, insbesondere über diejenigen, die demselben Vertriebshaus wie Mofos gehören - also praktisch alle pornografischen Websites im Internet, die am häufigsten angeklickt werden. ${ }^{5}$

Die in ihnen dargestellten Bilder offenbaren das kommerzielle Potenzial und den fast mythischen Status, den der Grenzsex in der Populärkultur nach wie vor hat, zumindest in Nordamerika, aber wahrscheinlich nicht nur dort. Der allgemeine Ton der Website, die Fotografien, die Videos von Männern in Uniform und nackten Frauen und sogar die sexuellen Positio-

3 https://www.bustle.com/articles/52629-border-patrol-sex-porn-site-sickeningly-depicts-rapes-of-migrant-women (letzter Zugriff: 23. September 2020).

4 https://www.dailydot.com/irl/border-patrol-porn-sexual-assault// (letzter Zugriff: 23. September 2020).

Die Vertriebsfirma heißt Mindgeek und kontrolliert YouPorn, Pornhub, Tube8, XTube, Red Tube und andere große Portale, die verschiedene Pornografie-Produktionen sammeln. 
nen fließen in ein Imaginäres ein, das auf erotische Weise die Ungleichheiten an der mexikanisch-amerikanischen Grenze reflektiert und neu verarbeitet. Die Machtverhältnisse werden durch die Kontrolle und den Missbrauch von Körpern deutlich gemacht, wie wir noch sehen werden. Auf der Website, die geschlossen wurde, befand sich auch eine Galerie mit Bildern der weiblichen Protagonistinnen der Videos, auf denen jeweils der Stempel „deportiert“ aufgedruckt war, um deutlich zu machen, dass sie, wie auch alle anderen sowie die weiteren Migrantinnen in den Videos, trotz der sexuellen Leistung, zurückgewiesen wurden.

Die Dynamik, die Handlung und die Charakterisierung der Figuren tragen dazu bei, die Unterschiede zwischen den Personen auf der einen und auf der anderen Seite der Grenze durch die stereotype Darstellung der Kategorien Legalität und Illegalität, Geschlecht und Ethnizität hervorzuheben. Die Protagonistinnen des sexuellen Austauschs sind am Ende alle, oder fast alle, bereit, mit den Agenten Sex zu haben, und werden im Allgemeinen so dargestellt, als seien sie ohne Agency, hier verstanden als Ausdruck von Wille, Wahl und Handlungsfähigkeit. Sie werden auf ihrer Reise in der Regel von Männern begleitet, bei denen es sich um ihre „Kojoten“ (so werden Menschenhändler an der Grenze zwischen Mexiko und den Vereinigten Staaten genannt) oder um andere männliche Personen handeln dürfte, die sich um sie kümmern sollen. Sobald sie in die Hände der Agenten gelangen, geht es der weiteren Handlung zufolge darum, dass sie entweder Opfer von Gewalt und Vergewaltigung werden oder einwilligen, Sex zu haben. Diese zweite Option ist dadurch motiviert, dass Migrantinnen den Geschlechtsverkehr als einen möglichen Ausweg aus der Abschiebung sehen, und basiert daher auf Erpressung.

Wie uns Saskia Sassen erinnert, schieben sowohl die allgemeine Meinung als auch die Einwanderungspolitik der Vereinigten Staaten „dem Individuum die ausschließliche Verantwortung für den Migrationsprozess zu und machen so das Individuum zu dem Ort [site im englischen Original], an dem die Ausübung staatlicher Autorität zum Ausdruck kommt“ (Sassen 1999, 17, Übersetzung der Autorin). Dieser individualisierende Ansatz macht nichts anderes, als die breiteren Strukturen zu ignorieren, die mit wirtschaftlichen und geopolitischen transnationalen und neokolonialen Prozessen zusammenhängen und in die sich die internationalen Migrationen einfügen.

Auf der anderen Seite betreibt die westliche Erzählung einen Reduktionismus, der Migrant*innen als Opfer oder Kriminelle kategorisiert, die es also entweder zu retten oder zu bestrafen gilt. Dieser Prozess verstärkt die 
Assoziation von Migrant*innen mit Gefahr, aber auch die Vorstellung, dass sie, egal in welche Kategorie sie fallen, irgendwie minderwertig und unfähig sind, für sich selbst zu sorgen. Das Problem besteht darin, dass die Definition von Migrantinnen als Opfer (der Konflikte, vor denen sie fliehen, des Menschenhandels, der Gewalt, der sie ausgesetzt sind) sie letztlich zu solchen macht und ihrer Handlungsfähigkeit beraubt, auch im Hinblick auf die Entscheidungen, die zu Beginn des Migrationsprojekts, während der Reise sowie bei den Verhandlungen, die auf dem Weg stattfinden, getroffen werden.

Fügt man diese beiden Aspekte zusammen, so kommt es definitiv dazu, dass lateinamerikanische Migrantinnen für ihre eigene Viktimisierung verantwortlich gemacht werden, und so produziert die Gewalt des Staates, welche die Gewalt der Grenze ist, an der Grenze zum Ausdruck kommt und sichtbar wird, Kategorien von Menschen und Körpern als illegal, ausbeutbar, vergewaltigbar.

Die Vergewaltigung kann als eine Technologie zur Reproduktion sozialer Grenzen verstanden werden, wie Luibhéid (2002) zeigt, und dazu beitragen, dass undokumentierte Frauen gemäß den nordamerikanischen Hierarchien von Geschlecht, Sexualität, Rasse und Klasse definiert werden. Man muss auch die Tatsache mitbedenken, dass undokumentierte Migrantinnen nicht über die Mittel verfügen, um anzuprangern und zu protestieren, und das bedeutet, dass die Vergewaltigung auch ein Mechanismus ist, welcher die Dichotomie legal/illegal verstärkt (ebd.).

Um auf Butlers Diskurs über die Repräsentation subalterner Subjekte zurückzukommen: Der Prozess der Entmenschlichung von denjenigen, die sich nicht selbst repräsentieren können, beseitigt das Risiko der Empathie, der Anerkennung von Identität und ermöglicht die Akzeptanz von Gewalt. Die von Border-Patrol-Agenten ausgeführte und dargestellte Vergewaltigung definiert und bekräftigt eine Schichtung von Geschlecht, Sexualität, Rasse und Klasse, relativ zum Rechtsstatus, und macht die Verkörperung der Grenze und die auf Körper ausgeübte Gewalt sichtbar. Laut dell'Agnese ist die Grenze also „eine gegenderte Landschaft, in der es im Kontakt mit einem Anderen, das traditionell als minderwertig und passiv konstruiert wird, immer noch möglich ist, über eine Vormachtstellung von Geschlecht und Rasse zu fantasieren, die in anderen Bereichen der nationalen Erzählung zunehmend fehlt“ (dell'Agnese 2005, 218, Übersetzung der Autorin).

In der Reihe Border Patrol Sex gibt es einige seltenere Fälle, in denen lateinamerikanische Migrantinnen bei der Bewältigung der Situation eine aktivere Rolle spielen und Sex als ein Mittel zur Verhandlung ihrer Situation 
und möglicherweise zur Vermeidung einer Abschiebung nutzen. Ich finde es interessant, diese Fälle getrennt von den anderen zu analysieren, denn obwohl sie nicht häufig vorkommen, unterbrechen sie meiner Meinung nach doch die diskursive Hegemonie und den Mechanismus der kanonischen Darstellung des Grenzübertritts. In diesen Situationen sind es die Migrantinnen, die, sobald sie von den Wachen angehalten werden, Sex als Tauschwährung gegen ihre Freiheit vorschlagen und, indem sie eine kontrollierende Rolle einnehmen, die Situation in den Griff bekommen und das Ende oft völlig umstoßen. In den allermeisten Fällen, in denen die Handlung in diese Richtung verläuft, gelingt es den Protagonistinnen zu entkommen, wobei sie manchmal die Waffe des Agenten stehlen, manchmal einfach nur seine Ablenkung ausnutzen. In solchen Fällen liegt der Unterschied in der Kontrolle, die sie ausüben können, sie bringen dann nämlich eine klare Fähigkeit zum Ausdruck, die Situation zu lesen, ihre Rolle zu verhandeln und sich der Zwickmühle zu entziehen. Zwar ändern sich die Machtmechanismen, die der Grenze zugrunde liegen, nicht, aber die Umkehrung der Situation untergräbt die in den vorhergehenden Fällen als selbstverständlich angenommene Assoziation zwischen Migrant*innen und Opfern und bringt die hegemoniale Erzählung aus dem Gleichgewicht. Die ironischen, geradezu komischen Aspekte der Situation erlauben es dem Agenten der Grenzpatrouille (und damit der von ihm vertretenen US-Regierung) bei solchen alternativen Endungen, „sein Gesicht zu wahren“, als wäre das Ganze nur ein Witz, eine unrealistische Situation. Abgesehen davon führt jedoch gerade die Möglichkeit eines unerwarteten Endes ein alternatives Potential in die Repräsentation der Grenze und der damit verbundenen Machtverhältnisse ein, das den Migrantinnen Handlungs- und Verhandlungsfähigkeit bezüglich der Situation zurückgibt und ein Definitionsvermögen bietet, was ihren Zustand betrifft, und sie folglich aus der Rolle passiver Opfer herausnimmt.

Einige Vorüberlegungen zu dieser Art der Repräsentation können jedenfalls nur bestätigen, dass die Pornifizierung der Grenze, indem sie die Machtverhältnisse explizit macht, dazu beiträgt, die Vorstellung von der Grenze als einem gewaltsamen Ort zu reproduzieren, an dem es den Sicherheitshütern des Westens erlaubt ist, auf die Körper unerwünschter Migrant*innen die Gewalt des Staates auszuüben, was durch eine angebliche intrinsische Überlegenheit gerechtfertigt wird.

Anstatt Schlussfolgerungen zu ziehen, glaube ich, dass sich aus dieser Analyse einige Fragen ergeben, die hauptsächlich die Möglichkeit einer alternativen und subversiven Darstellung der Grenze zwischen Mexiko und 
den Vereinigten Staaten sowie ihrer Überquerung betreffen, was sich auch mittels einer eingehenden Lektüre von border patrol pornography ergeben kann.

Wie bereits erwähnt, besteht eines der Probleme, die aus dem Prozess der Viktimisierung von Migrant*innen, vor allem aber in Bezug auf Frauen, resultieren, darin, dass dieser damit endet, dass ihre Handlungsfähigkeit verborgen und verleugnet wird, was zugleich durch den Prozess der Kriminalisierung geschieht, so dass die Individualitäten, Entscheidungen, Motivationen, Geschichten, Reisen, Wünsche und Erwartungen eines und einer jeden einzelnen von ihnen in einer sich vermindernden und verunglimpfenden kollektiven Definition verloren gehen. Natürlich reicht es nicht aus, Migrantinnen eine aktive Rolle in Pornovideos zuzugestehen, um den Diskurs und die Repräsentation ihrer Handlungsfähigkeit zu verändern, aber was ich in einer solchen Repräsentation sehe, ist etwas Neues, das einen Raum schafft, in dem es möglich wird, das typische Imaginäre von Migrantinnen als Opfer innerhalb eines standardisierten und heteronormativen Rahmens ethnischer und geschlechtsspezifischer Gewalt zu subvertieren. Diese Neuerung bietet nicht zuletzt die Möglichkeit, über ein alternatives Imaginäres nachzudenken und angesichts der Gewalt an Grenzen und deren Überwachung die Passivität nicht als etwas Selbstverständliches hinzunehmen. Interessant ist insbesondere die Möglichkeit, die Verbindung von Körpern, Grenzen und Gewalt und den Machtverhältnissen, die sich um die Grenze herum bilden und Kraft ausüben, zu entwirren, sowie die Idee einer Umkehrung des damit verbundenen Imaginären.

Eine mögliche Repräsentation, in der lateinamerikanische Migrantinnen ihren Willen zum Ausdruck bringen und situativ handeln, verdient es sicherlich, zusammen mit dem Potenzial eines Genres wie der Pornografie, das von der populären Geopolitik bisher fast vollständig ignoriert wurde, noch weiter untersucht zu werden. Die Grenze als Technologie der Kontrolle, auch durch die Disziplinierung von Körpern und die Ausübung von Gewalt, ist ein wesentlicher Forschungsgegenstand, um die Dynamik der Mobilität nicht nur zwischen Lateinamerika und den Vereinigten Staaten zu verstehen, sondern auch in einem Europa, das die Verwaltung der Grenzen und die Ausübung von Autorität - und damit auch von Gewalt - zunehmend an externe Institutionen und Akteure delegiert, sein Gewissen reinigt und das Problem beseitigt. 


\section{Literatur}

Aquino Moreschi, Alejandra. 2012. „Cruzando la frontera: Experiencias desde los márgenes“. Frontera Norte 24 (47): 7-34.

Balibar, Étienne. 2002. Politics and the Other Scene. London: Verso.

Butler, Judith. 2004. Precarious Life. The Power of Mourning and Violence. London und New York: Verso.

Coletti, Raffaella. 2018. „The Good Wife's (Geo)Politics Between Originality and Stereotypes: A New Wine or Just a New Bottle?" Geopolitics 23 (1): 50-66.

Cornelius, Wayne A. 2005. „Controlling ,Unwanted“ Immigration: Lessons from the United States, 1993-2004“. Journal of Ethnic and Migration Studies 31 (4): 775-794.

Dell'Agnese, Elena. 2005. „The US-Mexico Border in American Movies: A Political Geography Perspective“. Geopolitics 10 (2): 204-221.

Dell'Agnese, Elena. 2015. „Welcome to Tijuana': Popular Music on the US-Mexico Border“. Geopolitics 20 (1): 171-192.

Dell'Agnese, Elena, und Fabio Amato. 2016. „Perché studiare le migrazioni e la diaspora attraverso la cultura popolare“. Geotema 50: 5-9.

Dittmer, Jason, und Klaus Dodds. 2008. „Popular Geopolitics Past and Future: Fandom, Identities and Audience“. Geopolitics 13 (3): 437-457.

Donnan, Hastings, und Fiona Magowan. 2010. The Anthropology of Sex. London: Bloomsbury.

Gutiérrez, Ramón. 1996. „The Erotic Zone: Sexual Transgression on the US-Mexican Border". In Mapping Multiculturalism, herausgegeben von Avery F. Gordon und Christopher Newfield, 253-262. Minneapolis: University of Minnesota Press.

Holtzman, Linda. 200o. What Film, Television, and Popular Music Teach Us About Race, Class, Gender, and Sexual Orientation. New York und London: M.E. Sharpe.

Luibhéid, Eithne. 2002. Entry Denied: Controlling Sexuality at the Border. Minneapolis: University of Minnesota Press.

Menjívar, Cecilia. 2014. „Immigration Law Beyond Borders: Externalizing and Internalizing Border Controls in an Era of Securitization“. Annual Review of Law and Social Science 10: 353-369.

Paasonen, Susanna. 2011. Carnal Resonance: Affect and Online Pornography. Cambridge und London: MIT Press.

Parker, Noel, und Nick Vaughan-Williams. 2012. „Critical Border Studies: Broadening and Deepening the ,Lines in the Sand" Agenda“. Geopolitics 17 (4): 727-733.

Ó Tuathail, Gearóid. 1996. Critical Geopolitics. London: Routledge.

Rumford, Chris. 2012. „Towards a Multiperspectival Study of Borders“. Geopolitics 17 (4): 887-902. 
Sassen, Saskia. 1999. „Border Sovereignty: Immigration Policy Making Today“. In Immigration: a Civil Rights Issue for the Americas, herausgegeben von Susanne Jonas und Suzie Dod Thomas, 15-26. Wilmington: Rowan \& Littlefield.

Williams, Linda. 2004. „Porn Studies: Proliferating Pornographies On/Scene: An Introduction“. In Porn Studies, herausgegeben von L. Williams, 1-26. Durham und London: Duke University Press. 